\title{
A positive solution of a $p$-Laplace-like equation with critical growth
}

\author{
Zhou Yang $^{1 *}$, Di Geng ${ }^{1}$ and Huiwen Yan ${ }^{2}$
}

${ }^{\text {*Correspondence: }}$

yangzhou1975@yahoo.com.cn

${ }^{1}$ School of Mathematics Science, South China Normal University,

Guangzhou, 510631, China

Full list of author information is

available at the end of the article

\begin{abstract}
The existence of a positive solution of a $p$-Laplace-like equation with critical growth is established by the generalization to the concentration-compactness principle and the Sobolev inequality under some proper assumptions. Moreover, we achieve some regularity results of the solution.
\end{abstract}

MSC: $35 J 65$

Keywords: $p$-Laplace-like operator; critical growth; concentration-compactness principle; weakly continuity

\section{Introduction}

This paper is devoted to the existence of a positive solution of the following $p$-Laplace-like problem with critical growth:

$$
\begin{cases}-\operatorname{div}(a(\nabla u))=f(x, u), & x \in \Omega, \\ u=0, & x \in \partial \Omega,\end{cases}
$$

where $\Omega$ is a smooth bounded domain in $\mathbf{R}^{N}, 1<p<N$, and the functions $a, f$ satisfy some proper conditions, the details of which are described later.

There were many papers about the existence of the solution of $p$-Laplacian problems involving critical growth such as [1-6]. In them, $a(\xi)=|\xi|^{p-2} \xi$ and $f$ are some concrete functions with critical growth, which means that $f(x, u)|u|^{1-p^{\circ}}$ does not converge to zero as $u \rightarrow \infty$, where $p^{*}$ is the critical exponent, i.e., $p^{*}=N p /(N-p)$. The concentrationcompactness principle, which was built by Lions in $[7,8]$, plays an important role in achieving the existence of a nontrivial solution of the problems in them.

The authors proved the existence of a nonnegative and nontrivial solution for a Dirichlet problem for $p$-mean curvature operate with critical growth in [9], where $a(\xi)=(1+$ $\left.|\xi|^{2}\right)^{\frac{p-2}{2}} \xi, p \geq 2$, and $f$ is some concrete function involving a critical exponent. Since the function $a$ has an explicit form, the authors can use the concentration-compactness principle to achieve their results, too. But if $a$ is an abstract function in problem (1.1), then the problem becomes more complicated and interesting, and Lions' $\mathrm{C}-\mathrm{C}$ principle cannot be directly applied to it. Thanks to the generalization of the $\mathrm{C}-\mathrm{C}$ principle in [10], we can establish the existence of a nonnegative and nontrivial solution of equation (1.1) if we impose some proper conditions on the functions $a$ and $f$ and make more careful estimates. Moreover, we achieve some regularity result of the solution and prove the solution is positive under some proper assumptions. The results can be easily extended to a more general 
$p$-Laplace-like equation with critical growth and singular weights by the Caffarelli-KohnNirenberg inequality and the method in [11].

Recently, there have been some articles on stochastic partial differential equations (SPDEs) involving $p$-Laplace operator; see $[12,13]$. Some estimates and properties of the solution of the corresponding elliptic equations are important to the research on SPDEs. So, the results in this paper may be useful in the study of $p$-Laplace SPDEs with critical growth.

In this paper, we suppose that the potential $a: \mathbf{R}^{N} \rightarrow \mathbf{R}^{N}$ satisfies the following assumptions.

Let $A=A(\xi): \mathbf{R}^{N} \rightarrow \mathbf{R}$ be of continuous derivative with respect to $\xi$ with $a=\nabla_{\xi} A$ and satisfy the following conditions:

(A1) $A(0)=0$,

(A2) there are $p>1$ and $m \in[1, p]$, three positive constants $a_{1}, a_{2}$ and $a_{3}$ such that

$$
a_{1}|\xi|^{p} \leq|A(\xi)| \leq a_{2}|\xi|^{p}+a_{3}|\xi|^{m} \quad \text { for all } \xi \in \mathbf{R}^{N},
$$

(A3) $A(\xi)$ is strictly convex in $\xi$, that is, $2 A(\xi+\eta)<A(2 \xi)+A(2 \eta)$ for any $\xi \neq \eta \in \mathbf{R}^{N}$,

(A4) there exists a positive number $a_{4}$ such that $\lim _{|\xi| \rightarrow+\infty} a(\xi) \cdot \xi|\xi|^{-p}=a_{4}$.

We impose some assumptions on the critical nonlinear term $f(x, u): \bar{\Omega} \times \mathbf{R} \rightarrow \mathbf{R}$, which is continuous, as follows:

(B1) $f(x, 0)=0$,

(B2) there is a function $b(x) \in L^{\infty}(\Omega)$ such that

$$
\limsup _{u \rightarrow 0} \sup _{x \in \Omega}(f(x, u) u)|u|^{-p} \leq b(x)
$$

(B3) there are two positive numbers $c_{1}$ and $c_{2}$ such that

$$
\liminf _{u \rightarrow \infty} \inf _{x \in \Omega}(f(x, u) u)|u|^{-p^{*}} \geq c_{1}, \quad \limsup _{u \rightarrow \infty} \sup _{x \in \Omega}(f(x, u) u)|u|^{-p^{*}} \leq c_{2},
$$

(B4) denote $F(x, u)=\int_{0}^{u} f(x, s) d s$, which satisfies

$$
\liminf _{u \rightarrow \infty} \inf _{x \in \Omega}\left(f(x, u) u-p^{*} F(x, u)\right)|u|^{-p^{*}} \geq 0 .
$$

Moreover, we suppose $a$ and $f$ satisfy the next correlation.

(C1) there exists a $\beta>0$ such that

$$
\int_{\Omega}\left(p A(\nabla v)-b(x)|v|^{p}\right) d x \geq \beta \int_{\Omega} A(\nabla v) d x \quad \text { for any } v \in X
$$

where $X$ is $W_{0}^{1, p}(\Omega)$, i.e., the completion of $C_{0}^{\infty}(\Omega)$ with the norm $\|u\|_{X}=\left(\int_{\Omega}|\nabla u|^{p} d x\right)^{1 / p}$.

It is not difficult to see that both $a(\xi)=|\xi|^{p-2} \xi$ and $a(\xi)=\left(1+|\xi|^{2}\right)^{\frac{p-2}{2}} \xi$ with $p \geq 2$ satisfy (A1)-(A4), and the problems in [9] and [6] are concrete examples of problem (1.1). Moreover, we can consider some more generalized problem with a singular nonlinear term $f(x, u)$ with critical growth by the similar method in this paper and [11]. Then we can achieve more generalized results than those in [2] and [5], which will be considered 
in another paper. Since $a(z)$ is not an explicit function, we need to use the generalization of the C-C principle in [10] and more subtle estimates to study problem (1.1).

It is clear that the solution of problem (1.1) is the critical point of the variational functional

$$
I(u)=\int_{\Omega} A(\nabla u) d x-\int_{\Omega} F(x, u) d x .
$$

Moreover, $I(u)$ is continuous differentiable in $X$, and its Fréchet derivation is

$$
\left\langle I^{\prime}(u), v\right\rangle=\int_{\Omega} a(\nabla u) \cdot \nabla v d x-\int_{\Omega} f(x, u) v d x, \quad \forall v \in X .
$$

The first main result in this paper is

Theorem 1.1 Suppose problem (1.1) satisfies assumptions (A1)-(A4), (B1)-(B4) and (C1). Moreover, there exists a nontrivial $v_{0} \in X$ such that $v_{0} \geq 0$ and

(C2) $\sup _{t \geq 0} I\left(t v_{0}\right)<\left(\frac{1}{p}-\frac{1}{p^{*}}\right)(p \bar{S})^{\frac{p^{*}}{p^{*}-p}} c_{2}^{\frac{p}{p-p^{*}}}$, where $\bar{S}=\inf _{v \in X \backslash\{0\}}\|A(\nabla v)\|_{1}\|v\|_{p^{*}}^{-p}$, with $\|v\|_{q}^{q}=\int_{\Omega}|v|^{q} d x(q \geq 1)$.

Then problem (1.1) has a nonnegative and nontrivial solution.

Since the condition (C2) is difficult to check, we give another easily checked theorem.

Theorem 1.2 Assume conditions (A1)-(A4), (B1)-(B4) and (C1) are satisfied and

(A5) $p A(\xi) \geq a_{4}|\xi|^{p}$ for any $\xi \in \mathbf{R}^{N}$,

(B5) there exists a nonempty set $W \subset \Omega$ such that $F(x, u)-\frac{c_{2}}{p^{*}}|u|^{p^{*}} \geq 0$ for any $x \in W, u \in \mathbf{R}$,

(C3) $\lim _{\varepsilon \rightarrow 0^{+}}\left(\varepsilon^{\frac{(N-p) m}{(p-1) p}-N}+K_{2}(\phi)\right)\left(K_{1}(\psi)\right)^{-1}=0$, where $K_{1}(\psi)=\int_{0}^{\frac{1}{\varepsilon}} \Psi(r) d r$, $K_{2}(\phi)=\int_{0}^{\frac{1}{\varepsilon}} \Phi(r) d r$ with

$$
\begin{aligned}
& \Psi(r)=\psi\left[\left(\frac{\varepsilon^{-1}}{1+r^{p /(p-1)}}\right)^{\frac{N-p}{p}}\right] r^{N-1}, \quad \psi(r)=\min _{u \geq r, x \in W}\left(F(x, u)-\frac{c_{2}}{p^{*}}|u|^{p^{*}}\right), \\
& \Phi(r)=\phi\left[\left(\frac{\varepsilon^{-1}}{1+r^{p /(p-1)}}\right)^{\frac{N}{p}} r^{1 /(p-1)}\right] r^{N-1}, \quad \phi(r)=\max _{0 \leq|\xi| \leq r}\left(p A(\xi)-a_{4}|\xi|^{p}\right) .
\end{aligned}
$$

Then problem (1.1) has a nonnegative and nontrivial solution in X.

To establish the regularity of the solution $u$ and prove $u>0$ in $\Omega$, we need to impose stronger assumptions on the potential $a$ and the nonlinear $\operatorname{term} f$, which are as follows:

(D1) $A(\xi) \in C^{2}\left(\mathbf{R}^{N} \backslash\{0\}\right)$ with $a(0)=\mathbf{0}$ and there exist positive numbers $c$ and $C$, $\kappa \in[0,1]$ such that for any $\xi \in \mathbf{R}^{N} \backslash\{0\}$ and $\eta \in \mathbf{R}^{N}$,

$$
\sum_{i, j=1}^{N} \frac{\partial^{2} A}{\partial \xi_{i} \partial \xi_{j}} \eta_{i} \eta_{j} \geq c(\kappa+|\xi|)^{p-2}|\eta|^{2}, \quad \sum_{i, j=1}^{N}\left|\frac{\partial^{2} A}{\partial \xi_{i} \partial \xi_{j}}\right| \leq C(\kappa+|\xi|)^{p-2}
$$

(D2) $a$ admits the form $a(\xi)=g(|\xi|) \xi$. Moreover, $f(x, u) \geq 0$ for any $u \geq 0$. 
Theorem 1.3 Assume the assumptions in Theorem 1.1 or those in Theorem 1.2 hold, then there exists a constant $\alpha \in(0,1)$ such that the solution $u \in C^{1, \alpha}(\Omega)$ if assumption (D1) holds. Moreover, if assumption (D2) is satisfied, then $u>0$ in $\Omega$.

In Section 2, we will prove the main results. Some corollaries and examples are shown in Section 3.

\section{The proof of the main results}

First, we present the main tool in this paper - the generalized concentration-compactness principle, which is easily deduced from Theorem 2.1 in [10].

Lemma 2.1 Suppose assumptions (A1), (A2) and (A3) hold, $u_{n}$ weakly converges to $u$ in $X$ and $\mu_{n}=A\left(\nabla u_{n}\right) d x, v_{n}=\left|u_{n}\right|^{p^{*}} d x$ converge to $\mu, v$ weakly in the sense of measures, respectively.

Then there exist some at most countable set J, a family $\left\{x_{j} ; j \in J\right\}$ of distinct points in $\Omega$, and two families $\left\{v_{j} ; j \in J\right\},\left\{\mu_{j} ; j \in J\right\}$ of positive numbers such that

$$
\begin{aligned}
& v=|u|^{p^{*}} d x+\sum_{j \in J} v_{j} \delta_{x_{j}}, \quad \mu \geq A(\nabla u) d x+\sum_{j \in J} \mu_{j} \delta_{x_{j}}, \\
& \mu_{j} \geq \bar{S}\left(v_{j}\right)^{p / p^{*}} \quad(\forall j \in J),
\end{aligned}
$$

where $\delta_{x_{j}}$ denotes the Dirac measure at the point $x_{j}$.

Second, we deduce some properties of $a$ and $f$ by the similar method as in [11] or [6]. According to assumptions (A1)-(A4) and (B1)-(B4), we conclude that for any $\sigma>0, p \leq$ $k \leq p^{*}$, there exist some constants $C_{k, \sigma}, C_{\sigma}, C$ such that for any $\xi \in \mathbf{R}^{n}, x \in \Omega, u \in \mathbf{R}$,

$$
\begin{aligned}
& \left.\left|p A(\xi)-a_{4}\right| \xi\right|^{p}|+| a(\xi) \cdot \xi-\left.a_{4}|\xi|^{p}|\leq \sigma| \xi\right|^{p}+C_{\sigma}, \\
& \frac{c_{1}}{2 p^{*}}|u|^{p^{*}}-C \leq F(x, u) \leq \frac{b(x)+\sigma}{p}|u|^{p}+\frac{c_{2}+\sigma}{p^{*}}|u|^{p^{*}}+C_{k, \sigma}|u|^{k}, \\
& \frac{c_{1}}{2}|u|^{p^{*}}-C|u| \leq f(x, u) u \leq(b(x)+\sigma)|u|^{p}+\left(c_{2}+\sigma\right)|u|^{p^{*}}+C_{k, \sigma}|u|^{k}, \\
& f(x, u) u-p^{*} F(x, u) \geq-\sigma|u|^{p^{*}}-C_{\sigma}, \\
& \left(1+\frac{2 \sigma}{c_{1}}\right) f(x, u) u-p^{*} F(x, u) \geq-C_{\sigma} .
\end{aligned}
$$

To obtain a nonnegative solution, we first consider the following variational functional and its Fréchet derivation:

$$
\begin{aligned}
& \widetilde{I}(u)=\int_{\Omega} A(\nabla u) d x-\int_{\Omega} F\left(x, u^{+}\right) d x \\
& \left.\widetilde{I}^{\prime}(u), v\right\rangle=\int_{\Omega} a(\nabla u) \cdot \nabla v d x-\int_{\Omega} f\left(x, u^{+}\right) v d x, \quad \forall v \in X .
\end{aligned}
$$

It is clear that $\widetilde{I}(u)=I(u)$ if $u \geq 0$. Next, we deduce $\widetilde{I}(u)$ satisfies the geometrical result of the mountain pass theorem without the (PS) condition, i.e., 
Lemma 2.2 $\widetilde{I}(0)=0$ and there exist two constants $\alpha_{0}, \rho_{0}$ and a function $u_{1} \in X$ such that

$$
\left.\widetilde{I}(u)\right|_{\partial B_{\rho_{0}}(0)} \geq \alpha_{0}>0, \quad\left\|u_{1}\right\|_{X}>\rho_{0}, \quad \widetilde{I}\left(u_{1}\right) \leq 0,
$$

where $B_{\rho_{0}}(0)=\left\{u \in X,\|u\|_{X} \leq \rho_{0}\right\}, \partial B_{\rho_{0}}(0)$ denotes the boundary of $B_{\rho_{0}}(0)$.

Proof Let $u=0$ in (2.6), and we have $\widetilde{I}(0)=0$.

Choosing $k=p^{*}, \sigma=a_{1} \beta S / 2$ in (2.3) (where $S=\inf _{v \in X \backslash\{0\}}\|v\|_{X}^{p}\|v\|_{p^{\circ}}^{-p}$ is the best embedding constant from $X$ to $L^{p^{2}}(\Omega)$ ), and combining assumptions (C1), (A2), we have

$$
\widetilde{I}(u) \geq \int_{\Omega} A(\nabla u) d x-\int_{\Omega} \frac{2 b(x)+a_{1} \beta S}{2 p}\left|u^{+}\right|^{p}+C\left|u^{+}\right|^{p^{*}} d x \geq \frac{a_{1} \beta}{2 p}\|u\|_{X}^{p}-\bar{C}\|u\|_{X}^{p^{*}} .
$$

Hence, $\widetilde{I}(u)>0$ if $\|u\|_{X}$ is small enough. So, we have showed the existence of $\alpha_{0}$ and $\rho_{0}$ in (2.8).

Next, we construct $u_{1}$ satisfying (2.8). In fact, fixing a nonnegative and nontrivial function $u_{0}$, recalling assumption (A2) and (2.3), we deduce that there are positive constants $C_{1}, C_{2}, C_{3}$ such that

$$
\begin{aligned}
\widetilde{I}\left(t u_{0}\right) & \leq \int_{\Omega}\left(a_{2} t^{p}\left|\nabla u_{0}\right|^{p}+a_{3} t^{m}\left|\nabla u_{0}\right|^{m}\right) d x-\int_{\Omega}\left(\frac{c_{1} t^{p^{*}}}{2 p^{*}}\left|u^{+}\right|^{p^{*}}+C\right) d x \\
& \leq C_{1} t^{p}-C_{2} t^{p^{*}}+C_{3} .
\end{aligned}
$$

Hence, if $t$ is large enough, then we can set $u_{1}=t u_{0}$ satisfying (2.8).

According to the Ambrosetti-Rabinowitz mountain pass theorem without the (PS) condition, there exists a function sequence $\left\{u_{n}\right\}_{n=1}^{\infty} \subset X$ such that as $n \rightarrow \infty$,

$$
\widetilde{I}\left(u_{n}\right) \longrightarrow c_{0} \text { and } \widetilde{I}^{\prime}\left(u_{n}\right) \longrightarrow 0 \text { in } X^{*}, \quad \text { where } c_{0}=\inf _{\gamma \in \Gamma} \max _{u \in \gamma} \widetilde{I}(u) \geq \alpha_{0}>0 \text {, }
$$

$\Gamma$ denotes the class of continuous paths joining 0 to $u_{1}$ in $X, X^{\prime \prime}$ denotes the dual space of $X$.

Lemma 2.3 The sequence $\left\{u_{n}\right\}_{n=1}^{\infty}$ is bounded in $X$.

Proof Let $v=u_{n}$ in (2.7) and combine (2.6), (2.9). We see that as $n \rightarrow \infty$,

$$
\begin{aligned}
c_{0}+o(1)= & \widetilde{I}\left(u_{n}\right)-\delta\left\langle\widetilde{I}^{\prime}\left(u_{n}\right), u_{n}\right\rangle=(1-p \delta) \int_{\Omega} A\left(\nabla u_{n}\right) d x \\
& +\delta \int_{\Omega}\left[p A\left(\nabla u_{n}\right)-a\left(\nabla u_{n}\right) \cdot \nabla u_{n}\right]+\left[f\left(x, u_{n}^{+}\right) u_{n}-\frac{1}{\delta} F\left(x, u_{n}^{+}\right)\right] d x \\
\geq & \left(a_{1}(1-p \delta)-2 \delta \sigma\right)\left\|u_{n}\right\|_{X}^{p}-\bar{C}_{\sigma}, \quad \text { where } \delta=\frac{1}{p^{*}}\left(1+\frac{2 \sigma}{c_{1}}\right) .
\end{aligned}
$$

In the last inequality, we have used (2.2) and (2.5). If we fix a small enough $\sigma$ such that $a_{1}(1-p \delta)-2 \delta \sigma>0$ in the above inequality, then the conclusion in this lemma is obvious. 
As a result of the above preparations, we can prove Theorem 1.1.

Proof of Theorem 1.1 Since $\left\{u_{n}\right\}_{n=1}^{\infty}$ is bounded in $X$, it is easy to see there are a $u \in X$ and a subsequence of $\left\{u_{n}\right\}_{n=1}^{\infty}$, still denoted by itself, such that

$$
\begin{aligned}
& u_{n} \rightarrow u \quad \text { weakly in } X, \\
& u_{n} \rightarrow u \quad \text { a.e. in } \Omega \text { and strongly in } L^{q}(\Omega)\left(1 \leq q<p^{*}\right), \\
& f\left(x, u_{n}^{+}\right) \rightarrow f\left(x, u^{+}\right) \quad \text { weakly in } X^{*} .
\end{aligned}
$$

By the Helly theorem, there exist a subsequence, still denoted by itself, and two nonnegative measures $\mu$ and $v$ such that as $n \rightarrow \infty$,

$$
A\left(\nabla u_{n}\right) d x \stackrel{w}{\longrightarrow} \mu, \quad\left|u_{n}\right|^{p^{\circ}} d x \stackrel{w}{\longrightarrow} v \quad \text { weakly in the sense of measures. }
$$

Applying Lemma 2.1, we have the corresponding conclusions of Lemma 2.1.

Next, we establish the lower-bound of $v_{j}$ and $\mu_{j}$

$$
v_{j} \geq(p \bar{S})^{p^{n} /\left(p^{\prime \prime}-p\right)} c_{2}^{p^{n} /(p-p)^{n}}>0, \quad \mu_{j} \geq p^{p /\left(p^{n}-p\right)} \bar{S}^{p^{*} /\left(p^{*}-p\right)} c_{2}^{p /\left(p-p^{*}\right)} .
$$

Denote $\varphi$ as the cutoff function of the ball $B_{2}(0)$ in $\mathbf{R}^{N}$, i.e., which satisfies

$$
\begin{aligned}
& \varphi \in C_{0}^{\infty}\left(\mathbf{R}^{N}\right), \quad 0 \leq \varphi \leq 1, \\
& \varphi(x)=1 \quad \text { if } x \in B_{1}(0), \quad \varphi(x)=1 \quad \text { if } x \bar{\in} B_{2}(0) .
\end{aligned}
$$

Define $\varphi_{\varepsilon, j}=\varphi\left(\left(x-x_{j}\right) / \varepsilon\right.$ ) for every $\varepsilon>0$ and $x_{j} \in \mathbf{R}^{N}$ for any $j \in J$. Recalling Lemma 4 in [6], we have the following estimate:

$$
\begin{aligned}
\int_{\Omega}\left|u_{n} \nabla \varphi_{\varepsilon, j}\right|^{p} d x & \leq S^{-1}\left(\int_{\mathbf{R}^{N}}|\nabla \varphi|^{\frac{p^{*} p}{p^{2}-p}} d x\right)^{\frac{p^{*}-p}{p^{p}}} \int_{B\left(x_{j}, \varepsilon\right)}\left|\nabla u_{n}\right|^{p} d x \\
& \leq C \int_{B\left(x_{j}, \varepsilon\right)}\left|\nabla u_{n}\right|^{p} d x .
\end{aligned}
$$

Hence, $\left\{u_{n} \varphi_{\varepsilon, j}\right\}_{n=1}^{\infty}$ is still bounded in $X$ and the boundary is independent of $\varepsilon, j$.

Let $u=u_{n}, v=u_{n} \varphi_{\varepsilon, j}$ in (2.6) and combine (2.2), (2.4), (2.10), (2.15). Then we obtain as $n \rightarrow \infty$,

$$
\begin{aligned}
o(1)= & \left\langle\widetilde{I}^{\prime}\left(u_{n}\right), u_{n} \varphi_{\varepsilon, j}\right\rangle=\int_{\Omega} a\left(\nabla u_{n}\right) \cdot\left(\nabla u_{n} \varphi_{\varepsilon, j}+u_{n} \nabla \varphi_{\varepsilon, j}\right)-f\left(x, u_{n}^{+}\right) \varphi_{\varepsilon, j} u_{n} d x \\
\geq & p \int_{B_{\varepsilon / 2}\left(x_{j}\right)} A\left(\nabla u_{n}\right) d x-2 \sigma\left\|u_{n}\right\|_{X}^{p}-C_{\sigma} \operatorname{mes}\left(B_{\varepsilon}(0)\right) \\
& -\int_{B_{\varepsilon}\left(x_{j}\right)}\left(\left(c_{2}+\sigma\right)\left|u_{n}\right|^{p^{*}}+C_{\sigma}\right) d x \\
& -\left(\int_{\Omega}\left|a\left(\nabla u_{n}\right)\right|^{\frac{p}{p-1}} d x\right)^{\frac{p-1}{p}}\left(C \int_{B\left(x_{j}, \varepsilon\right)}\left|\nabla u_{n}\right|^{p} d x+o(1)\right) .
\end{aligned}
$$


In the above inequality, first letting $n \rightarrow \infty$, then taking $\varepsilon \rightarrow 0^{+}$, and finally taking $\sigma \rightarrow 0^{+}$, we deduce $p \mu_{j} \leq c_{2} v_{j}$. So, (2.1) implies (2.13). Since $v$ is a bounded measure, $J$ is at most finite.

In the following, we prove that $u \geq 0$, and it is the solution of problem (1.1). If $J$ is empty, then the proof is similar to that when $J$ is nonempty, which we omit. Next, we suppose $J$ is nonempty and denote $J=\{1,2, \ldots, m\}, \Omega_{\varepsilon}=\left\{x \in \Omega \mid \operatorname{dist}\left(x, x_{j}\right)>\varepsilon, \forall j \in J\right\}$. Fix a large enough $R$ and a sufficiently small $\varepsilon_{0}$ so that

$$
\bar{\Omega} \subset B_{R}(0), \quad B_{\varepsilon_{0}}\left(x_{i}\right) \cap B_{\varepsilon_{0}}\left(x_{j}\right)=\varnothing \quad \text { while } i \neq j \quad \text { and } \quad \bigcup_{j=1}^{m} B_{\varepsilon_{0}}\left(x_{j}\right) \subset B_{R}(0) \text {. }
$$

Define $\psi_{\varepsilon}(x)=\varphi(x / R)-\sum_{j=1}^{m} \varphi_{\varepsilon, j}(x)$ with $x \in \mathbf{R}^{N}, 0<\varepsilon \leq \varepsilon_{0}$. It is not difficult to deduce that $\left\{\psi_{\varepsilon} u_{n}\right\}_{n=1}^{\infty}$ is bounded in $X$ and the bound is independent of $\varepsilon$ from (2.15). According to (2.9) and (2.10), it is clear that as $n \rightarrow \infty$,

$$
\begin{aligned}
o(1) & =\left\langle\widetilde{I}^{\prime}\left(u_{n}\right)-\widetilde{I}^{\prime}(u),\left(u_{n}-u\right) \psi_{\varepsilon}\right\rangle \\
& =\int_{\Omega} J_{1}\left(u_{n}, u\right) \psi_{\varepsilon}+J_{2}\left(u_{n}, u\right)+J_{3}\left(u_{n}, u\right) d x,
\end{aligned}
$$

where $J_{1}\left(u_{n}, u\right)=\left(a\left(\nabla u_{n}\right)-a(\nabla u)\right) \cdot\left(\nabla u_{n}-\nabla u\right), J_{2}\left(u_{n}, u\right)=\left(a\left(\nabla u_{n}\right)-a(\nabla u)\right) \cdot \nabla \psi_{\varepsilon}\left(u_{n}-u\right)$ and $J_{3}\left(u_{n}, u\right)=\left(f\left(x, u^{+}\right)-f\left(x, u_{n}^{+}\right)\right) \psi_{\varepsilon}\left(u-u_{n}\right)$. Applying the method as in (2.16), we see that $\left|\int_{\Omega} J_{2}\left(u_{n}, u\right) d x\right|$ converges to 0 as $n \rightarrow \infty$. Moreover, the definition of $\psi_{\varepsilon}$ and (2.12) imply

$$
\lim _{n \rightarrow \infty} \int_{\Omega}\left|u_{n} \psi_{\varepsilon}\right|^{p^{*}} d x=\int_{\Omega}\left|\psi_{\varepsilon}\right|^{p^{*}} d v=\int_{\Omega}\left|u \psi_{\varepsilon}\right|^{p^{*}} d x
$$

Recalling (2.10), we see $u_{n} \psi_{\varepsilon} \rightarrow u \psi_{\varepsilon}$ in $L^{p^{*}}(\Omega)$. In view of (2.4), we obtain

$$
\begin{aligned}
& \left|\int_{\Omega} J_{3}\left(u_{n}, u\right) d x\right| \\
& \quad \leq\left(\int_{\Omega}\left(\left|f\left(x, u^{+}\right)\right|+\left|f\left(x, u_{n}^{+}\right)\right|\right)^{\frac{p^{*}}{p^{*}-1}} d x\right)^{\frac{p^{*}-1}{p^{*}}}\left\|\psi_{\varepsilon}\left(u_{n}-u\right)\right\|_{p^{*}} \rightarrow 0 .
\end{aligned}
$$

According to (2.17), (2.18), we deduce that $J_{1}\left(u_{n}, u\right)$ converges to 0 a.e. in $\Omega$ as $n \rightarrow \infty$, maybe extracting a subsequence. Since $A(\xi)$ is strictly convex, by the same method as [14], we claim $\nabla u_{n} \rightarrow \nabla u$ a.e. in $\Omega$, and there exists a subsequence, still denoted by itself, such that $a\left(\nabla u_{n}\right)$ weakly converges to $a(\nabla u)$ in $X^{*}$. Hence, (2.7), (2.9) and (2.11) imply that $\widetilde{I}^{\prime}\left(u_{n}\right)$ weakly converges to $\widetilde{I}^{\prime}(u)$ in $X^{*}$ and $\widetilde{I}^{\prime}(u)=0$. So, it is not difficult to see that $u \geq 0$ and $I^{\prime}(u)=\widetilde{I}^{\prime}(u)=0$, which means that $u$ is a weakly solution of equation (1.1).

Next, we prove $u$ is nontrivial, i.e., $u \neq 0$ if assumption (C2) holds. According to the definition of $\widetilde{I}(u)$ and the properties of $\left\{u_{n}\right\}_{n=1}^{\infty}$, we conclude that as $n \rightarrow \infty$,

$$
\begin{aligned}
& \left(o(1)+c_{0}-\widetilde{I}(u)\right)-\frac{1}{p^{*}} \sum_{j \in J}\left[\widetilde{I}^{\prime}\left(u_{n}\right), u_{n} \varphi_{\varepsilon, j}\right) \\
& =\int_{\Omega}\left(A\left(\nabla u_{n}\right)-A(\nabla u)\right) d x-\int_{\Omega}\left(F\left(x, u_{n}^{+}\right)-F\left(x, u^{+}\right)\right) d x
\end{aligned}
$$




$$
\begin{aligned}
& -\frac{1}{p^{*}} \sum_{j \in J} \int_{\Omega} a\left(\nabla u_{n}\right) \cdot \nabla\left(u_{n} \varphi_{\varepsilon, j}\right)-f\left(x, u_{n}^{+}\right) \varphi_{\varepsilon, j} u_{n} d x \\
= & K_{1}+K_{2}+\frac{1}{p^{*}} \sum_{j \in J}\left(p^{*} K_{3, j}+\left(p^{*}-p\right) \int_{\Omega} A\left(\nabla u_{n}\right) \varphi_{\varepsilon, j} d x+K_{4, j}-K_{5, j}+K_{6, j}\right),
\end{aligned}
$$

where $K_{3, j}=\int_{\Omega}\left(F\left(x, u^{+}\right)-A(\nabla u)\right) \varphi_{\varepsilon, j} d x$ converges to 0 as $\varepsilon \rightarrow 0^{+}$, and it has been proved in (2.16) that $K_{5, j}=\int_{\Omega} a\left(\nabla u_{n}\right) \cdot \nabla \varphi_{\varepsilon, j} u_{n} d x$ converges to 0 as $n \rightarrow \infty$. Moreover, as $n \rightarrow \infty$,

$$
\begin{aligned}
K_{1} & =\int_{\Omega}\left[A\left(\nabla u_{n}\right)-A(\nabla u)\right] \psi_{\varepsilon} d x \\
& =\int_{\Omega} \psi_{\varepsilon} d \mu-\int_{\Omega} \psi_{\varepsilon} A(\nabla u) d x+o(1) \geq o(1) \quad(\text { by }(2.1)), \\
K_{2} & =\int_{\Omega}\left[F\left(x, u_{n}^{+}\right)-F\left(x, u^{+}\right)\right] \psi_{\varepsilon} d x=o(1)
\end{aligned}
$$

(by the method similar to that in (2.18)),

$$
\begin{aligned}
K_{4, j} & =\int_{\Omega}\left[p A\left(\nabla u_{n}\right)-a\left(\nabla u_{n}\right) \cdot \nabla u_{n}\right] \varphi_{\varepsilon, j} d x \\
& \geq-2 \sigma\left\|u_{n}\right\|_{X}^{p}-C_{\sigma} \operatorname{mes}\left(B_{\varepsilon}(0)\right) \quad(\text { by }(2.2)), \\
K_{6, j} & =\int_{\Omega}\left[f\left(x, u_{n}^{+}\right) u_{n}-p^{*} F\left(x, u_{n}^{+}\right)\right] \varphi_{\varepsilon, j} d x \\
& \geq-\sigma\left\|u_{n}\right\|_{p^{\prime \prime}}^{p^{\prime \prime}}-C_{\sigma} \operatorname{mes}\left(B_{\varepsilon}(0)\right) \quad(\text { by }(2.5)) .
\end{aligned}
$$

Combining (2.12), (2.13) and the above inequalities and equalities, firstly taking $n \rightarrow \infty$, then taking $\varepsilon \rightarrow 0^{+}$, and finally taking $\sigma \rightarrow 0^{+}$in (2.19), we have

$$
c_{0}-I(u)=c_{0}-\widetilde{I}(u) \geq \frac{p^{*}-p}{p^{*}} \mu_{j} \geq\left(\frac{1}{p}-\frac{1}{p^{*}}\right)(p \bar{S})^{\frac{p^{n}}{p^{*}-p}} c_{2}^{\frac{p}{p-p^{*}}} .
$$

According to the definition of $c_{0}$ and assumption (C2), we have $I(u)<0$, which means $u \neq 0$.

Before proving Theorem 1.2, we need to introduce the function family $\left\{v_{\varepsilon}\right\}$ which approximates the best embedding constant $S$ from $X$ to $L^{p}(\Omega)$. Without loss of generalization, we suppose $B_{2}(0) \subset \Omega, 0<\varepsilon<1$. Denote

$$
U=\frac{1}{\left(1+|x|^{p /(p-1)}\right)^{(N-p) / p}}, \quad U_{\varepsilon}=\varepsilon^{\frac{p-N}{p}} U\left(\frac{x}{\varepsilon}\right), \quad u_{\varepsilon}=U_{\varepsilon} \varphi, \quad v_{\varepsilon}=u_{\varepsilon}\left\|u_{\varepsilon}\right\|_{p^{\prime \prime}}^{-1}
$$

where $\varphi$ is defined in (2.14), and $U$ is the extremal function reaching $S$. It is easy to check that as $\varepsilon \rightarrow 0^{+}$,

$$
\begin{aligned}
& \int_{\mathbf{R}^{N} \backslash B_{1}(0)}\left|U_{\varepsilon}\right|^{p^{*}} d x=O\left(\varepsilon^{\frac{N}{p-1}}\right), \quad \int_{\mathbf{R}^{N} \backslash B_{1}(0)}\left|\nabla U_{\varepsilon}\right|^{p} d x=O\left(\varepsilon^{\frac{N-p}{p-1}}\right), \\
& \int_{B_{2}(0) \backslash B_{1}(0)}\left|U_{\varepsilon}\right|^{m} d x=\int_{B_{2}(0) \backslash B_{1}(0)}\left|\nabla U_{\varepsilon}\right|^{m} d x=O\left(\varepsilon^{\frac{(N-p) m}{(p-1) p}}\right), \\
& \left\|v_{\varepsilon}\right\|_{p^{*}}^{p^{*}}=1, \quad\left\|v_{\varepsilon}\right\|_{p}^{p}=o(1), \quad\left\|v_{\varepsilon}\right\|_{X}^{p}=S+O\left(\varepsilon^{(N-p) /(p-1)}\right) .
\end{aligned}
$$


As a result of the preparations, we can prove Theorem 1.2 as follows.

Proof of Theorem 1.2 Without loss of generalization, we suppose $B_{2}(0) \subset W$. For convenience, let $A$ and $B$ denote some positive constants which may be different in different places.

Applying the method in the proof of Lemma 2.2, we deduce that $I\left(t v_{\varepsilon}\right) \rightarrow-\infty$ as $t \rightarrow$ $+\infty$, which implies there exists a $t_{\varepsilon} \geq 0$ such that $I\left(t_{\varepsilon} v_{\varepsilon}\right)=\sup _{t \geq 0} I\left(t v_{\varepsilon}\right)$ and

$$
\left.\frac{d}{d t} I\left(t v_{\varepsilon}\right)\right|_{t=t_{\varepsilon}}=\left\langle I^{\prime}\left(t_{\varepsilon} v_{\varepsilon}\right), v_{\varepsilon}\right\rangle=\int_{\Omega} a\left(t_{\varepsilon} \nabla v_{\varepsilon}\right) \cdot \nabla v_{\varepsilon} d x-\int_{\Omega} f\left(x, t_{\varepsilon} v_{\varepsilon}\right) v_{\varepsilon} d x=0 .
$$

Let $\sigma=1$ and $k=p$ in (2.4), combining (2.23), (A1), (A3) and (A5), we obtain

$$
\begin{aligned}
a_{4} t_{\varepsilon}^{p}\left\|v_{\varepsilon}\right\|_{X}^{p} & \leq \int_{\Omega} A\left(t_{\varepsilon} \nabla v_{\varepsilon}\right) d x \leq t_{\varepsilon} \int_{\Omega} a\left(t_{\varepsilon} \nabla v_{\varepsilon}\right) \cdot \nabla v_{\varepsilon} d x \\
& \leq C\left(t_{\varepsilon}^{p}\left\|v_{\varepsilon}\right\|_{p}^{p}+t_{\varepsilon}^{p^{*}}\left\|v_{\varepsilon}\right\|_{p^{2}}^{p^{*}}\right) .
\end{aligned}
$$

Recalling (2.22), we see that $t_{\varepsilon}$ is positive and bounded away from zero as $\varepsilon \rightarrow 0^{+}$. Moreover, according to assumption (A2) and (2.4), we infer

$$
\begin{aligned}
C\left(t_{\varepsilon}^{p}\left\|v_{\varepsilon}\right\|_{X}^{p}+1\right) & \geq t_{\varepsilon} \int_{\Omega} a\left(t_{\varepsilon} \nabla v_{\varepsilon}\right) \cdot \nabla v_{\varepsilon} d x=\int_{\Omega} f\left(x, t_{\varepsilon} v_{\varepsilon}\right) t_{\varepsilon} v_{\varepsilon} d x \\
& \geq \frac{c_{1}}{2} t_{\varepsilon}^{p^{*}}\left\|v_{\varepsilon}\right\|_{p^{p^{*}}}^{p^{*}}-C .
\end{aligned}
$$

Hence, (2.22) implies $t_{\varepsilon}$ is bounded, and the bound is independent of $\varepsilon$.

Set

$$
h_{0}(t)=\frac{a_{4}}{p} t^{p}\left\|v_{\varepsilon}\right\|_{X}^{p}-\frac{c_{2}}{p^{*}} t^{p^{*}}\left\|v_{\varepsilon}\right\|_{p^{*}}^{p^{*}}
$$

In view of (2.22), we have

$$
\max _{t \geq 0} h_{0}(t)=\left(\frac{1}{p}-\frac{1}{p^{*}}\right)\left(\frac{a_{4}\left\|v_{\varepsilon}\right\|_{X}^{p}}{\left\|v_{\varepsilon}\right\|_{p^{*}}^{p}}\right)^{\frac{p^{*}}{p^{*}-p}} c_{2}^{\frac{p}{p-p^{*}}}=\left(\frac{1}{p}-\frac{1}{p^{*}}\right)\left(a_{4} S\right)^{\frac{p^{*}}{p^{*}-p}} c_{2}^{\frac{p}{p-p^{*}}}+O\left(\varepsilon^{\frac{N-p}{p^{-1}}}\right) .
$$

It is noted that $\phi(r)$ is increasing with $\phi(0)=0$ according to assumption (C3). Hence, we deduce

$$
\begin{aligned}
\sup _{t \geq 0} I\left(t v_{\varepsilon}\right)= & I\left(t_{\varepsilon} v_{\varepsilon}\right) \leq h_{0}\left(t_{\varepsilon}\right)+\frac{1}{p} \int_{\Omega} \phi\left(t_{\varepsilon}\left|\nabla v_{\varepsilon}\right|\right) d x-\int_{\Omega} \psi\left(t_{\varepsilon} v_{\varepsilon}\right) d x \\
\leq & \left(\frac{1}{p}-\frac{1}{p^{*}}\right)\left(a_{4} S\right)^{\frac{p^{\circ}}{p^{*}-p}} c_{2}^{\frac{p}{p-p^{*}}} \\
& +O\left(\varepsilon^{\frac{N-p}{p-1}}\right)+\frac{1}{p} \int_{\Omega} \phi\left(t_{\varepsilon}\left|\nabla v_{\varepsilon}\right|\right) d x-\int_{\Omega} \psi\left(t_{\varepsilon} v_{\varepsilon}\right) d x .
\end{aligned}
$$

Next, we handle the last two terms on the right-hand side of (2.24). Since $\phi(r)$ is nonnegative and increasing, we can utilize the properties of $v_{\varepsilon}$ and $t_{\varepsilon}$ to calculate

$$
0 \leq \int_{\Omega} \phi\left(t_{\varepsilon}\left|\nabla v_{\varepsilon}\right|\right) d x \leq \int_{B_{1}(0)} \phi\left(A\left|\nabla U_{\varepsilon}\right|\right) d x+\int_{B_{2}(0) \backslash B_{1}(0)} \phi\left(A\left|\nabla u_{\varepsilon}\right|\right) d x
$$




$$
\begin{aligned}
\int_{B_{1}(0)} \phi\left(A\left|\nabla U_{\varepsilon}\right|\right) d x & \leq \int_{B_{1 / \varepsilon}(0)} \varepsilon^{N} \phi\left(A \varepsilon^{\frac{-N}{p}}|\nabla U|\right) d x \\
& \leq B \varepsilon^{N}\left(\int_{0}^{\varepsilon^{-1}}+\int_{\varepsilon^{-1}}^{A \varepsilon^{-1}}\right) \Phi(r) d r
\end{aligned}
$$

where $\Phi(r)$ is defined in assumption (C3). Without loss of generalization, suppose $A>1$ and $0<\varepsilon<1$. According to the definition of $\phi$ and assumption (A2), it is clear that $0 \leq$ $\phi(r) \leq a_{2} r^{p}+a_{3} r^{m}$, then we have

$$
B \varepsilon^{N} \int_{\varepsilon^{-1}}^{A \varepsilon^{-1}} \Phi(r) d r=\int_{B_{2}(0) \backslash B_{1}(0)} \phi\left(A\left|\nabla u_{\varepsilon}\right|\right) d x=O\left(\varepsilon^{\frac{(N-p) m}{(p-1) p}}\right) .
$$

We have used (2.20) and (2.21) in the last equality. Furthermore, (2.3) and the definition of $\psi(u)$ imply that $\psi(u) \leq B\left(|u|^{p}+|u|^{p^{*}}\right)$. Repeating the above argument, we obtain

$$
\int_{\Omega} \psi\left(t_{\varepsilon} v_{\varepsilon}\right) d x \geq B \varepsilon^{N} \int_{0}^{\varepsilon^{-1}} \Psi(r) d r+O\left(\varepsilon^{\frac{N-p}{p-1}}\right)
$$

Remembering assumption (A5), we see $p \bar{S} \geq a_{4} S$. Combining (2.24)-(2.28), we obtain

$$
\sup _{t \geq 0} I\left(t v_{\varepsilon}\right) \leq\left(\frac{1}{p}-\frac{1}{p^{*}}\right)(p \bar{S})^{\frac{p^{*}}{p^{*}-p}} c_{2}^{\frac{p}{p-p^{*}}}-\varepsilon^{N}\left(B K_{1}(\psi)-\bar{B} K_{2}(\phi)\right)+O\left(\varepsilon^{\frac{(N-p) m}{(p-1) p}}\right) .
$$

So, assumption (C3) implies that assumption (C2) holds if we choose $\varepsilon$ small enough, and the conclusion in Theorem 1.2 follows from Theorem 1.1.

Proof of Theorem 1.3 We firstly prove $u \in L^{\infty}(\Omega)$ by the Moser iteration. Since the problem involves critical growth, we need some preparation before making the Moser iteration. Set $\eta(t) \in C(\mathbf{R})$ and

$$
\eta(t)=\left\{\begin{array}{ll}
\operatorname{sgn}(t)|t|^{k} & \text { if }|t| \leq M, \\
\text { linear } & \text { if }|t| \geq M,
\end{array} \quad \text { where } k>1, M>1, \quad \xi(t)=\int_{0}^{t}\left(\eta^{\prime}(s)\right)^{p} d s\right.
$$

It is not difficult to check that $\eta^{\prime}(t)>0$ and $\xi(u) \in X$ if $u \in X$, and for any $t \in \mathbf{R}$,

$$
\begin{aligned}
& |\xi(t)| \leq k|\eta(t)|\left(\eta^{\prime}(t)\right)^{p-1}, \quad \eta^{\prime}(t) \leq k(1+|\eta(t)|), \\
& |\xi(t)||t|^{p-1} \leq k^{p}|\eta(t)|^{p} .
\end{aligned}
$$

Let $v(x)=\xi(u(x)) \psi^{p}(x)$ in (1.3), where $\psi(x)=\varphi\left(\left(x-x_{0}\right) / R_{0}\right)$ and $\varphi$ is defined in (2.14), $x_{0} \subset \Omega, R_{0}>0$. Denote $D=B_{2 R_{0}}\left(x_{0}\right) \cap \Omega, E=B_{R_{0}}\left(x_{0}\right) \cap \Omega$, then we compute

$$
\begin{aligned}
& \int_{\Omega} a(\nabla u) \cdot \nabla u \xi^{\prime}(u) \psi^{p} d x+p \int_{\Omega} a(\nabla u) \cdot \nabla \psi \xi(u) \psi^{p-1} d x \\
& \quad=\int_{\Omega} f(x, u) \xi(u) \psi^{p} d x .
\end{aligned}
$$


Denote the first term and the second term on the left-hand side and on the right-hand side of (2.30) as $J_{1}$ and $J_{2}, J_{3}$, respectively. Now, we estimate them as follows:

$$
\begin{aligned}
J_{1} & \geq a_{1} \int_{\Omega}|\nabla u|^{p} \psi^{p} \xi^{\prime}(u) d x=a_{1} \int_{\Omega}|\nabla \eta(u)|^{p} \psi^{p} d x \\
& =a_{1} \int_{D}\left(|\nabla(\eta(u) \psi)|^{p}-|\eta(u) \nabla \psi|^{p}\right) d x, \\
J_{2} & \geq-p C \int_{\Omega}\left(|\nabla u|^{p-1}+|\nabla u|^{m-1}\right)|\xi(u)||\nabla \psi| \psi^{p-1} d x \\
& \geq-p k C \int_{D}\left(\left|\eta^{\prime}(u) \nabla u\right|^{p-1}+\left|\eta^{\prime}(u)\right|^{p-1}|\nabla u|^{m-1}\right)|\eta(u)||\nabla \psi| \psi^{p-1} d x \quad \text { (by (2.29)) } \\
& \geq-\sigma \int_{D}|\nabla \eta(u)|^{p} \psi^{p} d x-\sigma J_{4}-C_{\sigma} k^{p} \int_{D}|\eta(u)|^{p}|\nabla \psi|^{p} d x,
\end{aligned}
$$

where $\sigma$ is a positive number defined later and

$$
\begin{aligned}
J_{4} & =\int_{D}\left|\eta^{\prime}(u)\right|^{p}|\nabla u|^{\frac{m-1}{p^{-1}} p} \psi^{p} d x \leq k^{p} \int_{D}(1+|\eta(u)|)^{p}\left(1+|\nabla u|^{p}\right) \psi^{p} d x \quad \text { (by (2.29)) } \\
& \leq \int_{D}|\nabla \eta(u)|^{p} \psi^{p} d x+k^{p} \int_{D}|\eta(u)|^{p} \psi^{p} d x+C k^{p}, \\
J_{3} & \leq C \int_{\Omega}\left(|u|^{p^{*}-1}+1\right) \xi(u) \psi^{p} d x \\
& \leq C k^{p} \int_{\Omega}|u|^{p^{*}-p}|\eta(u)|^{p} \psi^{p}+|\eta(u)|(1+|\eta(u)|)^{p-1} \psi^{p} d x \quad \text { (by (2.29)) } \\
& \leq C k^{p}\left(\int_{D}|\eta(u) \psi|^{p^{*}} d x\right)^{\frac{p}{p^{p}}}\left(\left(\int_{D}|u|^{p^{*}} d x\right)^{\frac{p^{*}-p}{p^{p}}}+\operatorname{mes}(D)^{\frac{p^{p^{*}-p}}{p^{p}}}\right)+C k^{p} .
\end{aligned}
$$

Set $k=k_{0}=p^{*} / p$ in view of (2.30) and the above equalities. If we firstly fix a small enough $\sigma$, then a small enough $R_{0}$, then we can obtain

$$
\left(\int_{E}|\eta(u)|^{p^{\circ}} d x\right)^{p / p^{*}} \leq C \int_{E}|\nabla \eta(u)|^{p} d x \leq C \int_{D}|\eta(u)|^{p} d x+C,
$$

where $C$ is a constant independent of $M$. Taking $M \rightarrow+\infty$, then we deduce $u \in L^{k_{0} p^{\prime \prime}}(E)$. Applying a simple covering argument, we achieve that $u \in L^{k_{0} p^{*}}(\Omega)$. Finally, repeating the same argument, we derive that $u \in L^{p^{*}+p}(\Omega)$. As a result of the preparations, we can use the Moser iteration to prove $u \in L^{\infty}(\Omega)$. Let $v(x)=\xi(u(x))$ in (1.3), and we calculate

$$
\begin{aligned}
a_{1} \int_{\Omega}|\nabla \eta(u)|^{p} d x & \leq \int_{\Omega} a(\nabla u) \cdot \nabla u \xi^{\prime}(u) d x=\int_{\Omega} f(x, u) \xi(u) d x \\
& \leq C \int_{\Omega}\left(|u|^{p^{*}-1}+1\right) \xi(u) d x \\
& \leq C k^{p} \int_{\Omega}\left(|u|^{p^{*}-p}+1\right)|\eta(u)|^{p}+1 d x \quad(\text { by }(2.29)) \\
& \leq C k^{p}\left(\int_{\Omega}|\eta(u)|^{\left(p^{*}+p\right) / 2} d x\right)^{\frac{2 p}{p^{p}+p}}\left(\left(\int_{\Omega}|u|^{p^{*}+p} d x\right)^{\frac{p^{\circ}-p}{p^{*}+p}}+1\right)+C k^{p},
\end{aligned}
$$


where $C$ is a constant independent of $M$ and $k$. If we set $\lambda=\left(p^{*}+p\right) /\left(2 p^{*}\right)$, then the above inequality implies

$$
\|u\|_{k p^{*}} \leq C^{1 / k p} k^{1 / k}\left(1+\|u\|_{\lambda k p^{*}}^{k p}\right)^{1 /(k p)} .
$$

Thus, $u \in L^{\infty}(\Omega)$ follows from the standard Moser iteration method.

Applying Theorem 1 in [15], we see that there exists a constant $\alpha \in(0,1)$ such that $u \in C^{1, \alpha}(\Omega)$. If we rewrite $f(x, u)$ as $(f(x, u) / u) u$, then $f(x, u) / u \in L^{\infty}(\Omega)$ with $f(x, u) / u \geq 0$. Employing Theorem 6 in [16], it is obvious that $u>0$ in $\Omega$.

\section{Some corollaries and examples}

In this section, we firstly consider when (C3) is true through analyzing $K_{1}(\psi)$ and $K_{2}(\phi)$, then we give some concrete examples and corollaries.

Firstly, we analyze the effect of $a(z)$ to $K_{2}(\phi)$ :

Lemma 3.1 Suppose a(z) satisfies assumptions (A1)-(A5) and

(A6) There exist positive numbers $m_{1}, m_{2}, A, B$ such that $\phi(r) \leq B r^{m_{1}}$ for any $0 \leq r \leq A$ and $\phi(r) \leq B r^{m_{2}}$ for any $r \geq A$.

Then $K_{2}(\phi)=O\left(\lambda_{2}(\varepsilon)+\lambda_{3}(\varepsilon)\right)$ as $\varepsilon \rightarrow 0^{+}$, where $K_{2}(\phi)$ is defined in Theorem 1.2 and

$$
\lambda_{2}(\varepsilon)=\left\{\begin{array}{ll}
\varepsilon^{\frac{(N-p) m_{1}}{(p-1) p}-N} & \text { if } m_{1}<\frac{N(p-1)}{N-1}, \\
\varepsilon^{\frac{-m_{1} N}{p}}|\ln \varepsilon| & \text { if } m_{1}=\frac{N(p-1)}{N-1}, \\
\varepsilon^{\frac{N^{2}(1-p)}{(N-1) p}} & \text { if } m_{1}>\frac{N(p-1)}{N-1},
\end{array} \quad \lambda_{3}(\varepsilon)= \begin{cases}\varepsilon^{\frac{N^{2}(1-p)}{(N-1) p}} & \text { if } m_{2}<\frac{N(p-1)}{N-1}, \\
\varepsilon^{\frac{-m_{2} N}{p}}|\ln \varepsilon| & \text { if } m_{2}=\frac{N(p-1)}{N-1}, \\
\varepsilon^{\frac{-m_{2} N}{p}} & \text { if } m_{2}>\frac{N(p-1)}{N-1} .\end{cases}\right.
$$

Proof Repeating the argument similar to (2.26), we compute

$$
\begin{aligned}
0 \leq & K_{2}(\phi) \leq B \int_{0}^{A \varepsilon^{(p-1) N / p}} \varepsilon^{\frac{-m_{1} N}{p}} r^{N+\frac{m_{1}}{p-1}-1} d r+B \int_{A \varepsilon}^{\varepsilon^{-1}} \frac{-\frac{N(p-1)}{(N-1) p}}{\frac{-m_{1} N}{p}} r^{N-\frac{N-1}{p-1} m_{1}-1} d r \\
& +B \int_{A \varepsilon}^{1} \varepsilon^{\frac{-m_{2} N}{p}} r^{N+\frac{m_{2}}{p-1}-1} d r+B \int_{1}^{A \varepsilon^{-\frac{N(p-1)}{(N-1) p}}} \varepsilon^{\frac{-m_{2} N}{p}} r^{N-\frac{N-1}{p-1} m_{2}-1} d r \\
= & \lambda_{2}(\varepsilon)+\lambda_{3}(\varepsilon) .
\end{aligned}
$$

Secondly, we analyze how $\psi(u)$ affects $K_{1}(\psi)$. The proof is similar to the above, and we omit it.

Lemma 3.2 Suppose $\psi(u)$ defined in Theorem 1.2 satisfies

(B6) There are positive numbers $A, B$ and $q \geq p$ such that $\psi(u) \geq B|u|^{q}$ when $0 \leq u \leq A$. Then we have $K_{1}(\psi) \geq \lambda_{4}(\varepsilon)$ as $\varepsilon \rightarrow 0^{+}$, where

$$
\lambda_{4}(\varepsilon)= \begin{cases}C \varepsilon^{\frac{(N-p) q}{(p-1) p}-N} & \text { if } q<\frac{N(p-1)}{N-p}, \\ C \varepsilon^{\frac{N(1-p)}{p}}|\ln \varepsilon| & \text { if } q=\frac{N(p-1)}{N-p}, \quad C \text { is a positive constant. } \\ C \varepsilon^{\frac{N(1-p)}{p}} & \text { if } q>\frac{N(p-1)}{N-p},\end{cases}
$$

Lemma 3.3 Suppose $\psi(u)$ defined in Theorem 1.2 satisfies 
(B7) There exist positive numbers $A, B$ and $q<p^{*}$ such that $\psi(u) \geq B|u|^{q}$ if $u \geq A$.

Then $K_{1}(\psi) \geq \lambda_{5}(\varepsilon)$ as $\varepsilon \rightarrow 0^{+}$, where

$$
\lambda_{5}(\varepsilon)= \begin{cases}C \varepsilon^{\frac{N(1-p)}{p}} & \text { if } q<\frac{N(p-1)}{N-p}, \\ C \varepsilon^{\frac{N(1-p)}{p}}|\ln \varepsilon| & \text { if } q=\frac{N(p-1)}{N-p}, \quad C \text { is a positive constant. } \\ C \varepsilon^{\frac{(p-N) q}{p}} & \text { if } q>\frac{N(p-1)}{N-p},\end{cases}
$$

In the following, we can utilize Theorem 1.2 and Lemmas 3.1-3.3 to prove the following results about some concrete problems. The proof is trivial and we omit it.

Corollary 3.4 Assume $a(\xi)=\left(1+|\xi|^{2}\right)^{\frac{p-2}{2}} \xi, p \geq 2$ in problem (1.1), and assumptions (B1)(B5), (C1) hold, $\psi(r)$ defined in assumption (B5) satisfies

(B8) $\lim _{\varepsilon \rightarrow 0^{+}} \frac{K_{1}(\psi)}{\lambda_{6}(\varepsilon)}=+\infty, \quad$ where $\lambda_{6}(\varepsilon)= \begin{cases}\varepsilon^{-N+\frac{2(N-p)}{p(p-1)}} & \text { if } p>3-\frac{2}{N}, \\ \varepsilon^{-N+\frac{2(N-p)}{p(p-1)}}|\ln \varepsilon| & \text { if } p=3-\frac{2}{N} \\ \varepsilon^{-\frac{N^{2}(p-1)}{(N-1) p}} & \text { if } p<3-\frac{2}{N} .\end{cases}$

Then problem (1.1) possesses a nontrivial solution.

Proof Take $m_{1}=2$ and $m_{2}=p-2$ in Lemma 3.1, and we can deduce the conclusion.

Example 3.1 Next, we consider the following equation:

$$
\begin{cases}-\operatorname{div}\left(\left(1+|\nabla u|^{2}\right)^{\frac{p-2}{2}} \nabla u\right)=c|u|^{p^{\prime \prime}-2} u+k(x)|u|^{q-2} u+g(x, u), & x \in \Omega, \\ u=0, & x \in \partial \Omega .\end{cases}
$$

Corollary 3.5 Suppose the parameters $2 \leq p<q<p^{*}$ and $c>0$, the functions $k(x) \in C(\bar{\Omega})$ with $k(x) \geq k^{*}>0$, and $g(x, u) \in C(\bar{\Omega} \times \mathbf{R})$ with $g(x, 0)=0, g(x, u) u \geq 0$. Moreover,

$$
\begin{aligned}
& q>\left\{\begin{array}{ll}
p^{*}-\frac{N}{N-1} & \text { if } 2 \leq p \leq 3-\frac{2}{N}, \\
p^{*}-\frac{2}{p-1} & \text { if } p \geq 3-\frac{2}{N},
\end{array} \quad \lim _{u \rightarrow \infty} \sup _{x \in \Omega} \frac{|g(x, u)|}{|u|^{p^{*}-1}}=0,\right. \\
& \limsup \sup _{u \rightarrow 0} \frac{g(x, u)}{|u|^{p-2} u}<S .
\end{aligned}
$$

Then problem (3.1) possesses a positive solution in $C^{1, \alpha}(\Omega)(\alpha \in(0,1))$.

Example 3.2 Next, we consider the following equation:

$$
\begin{cases}-\operatorname{div}\left(|\nabla u|^{p-2} \nabla u+\frac{|\nabla u|^{\alpha+p-2}}{1+|\nabla u|^{p}} \nabla u\right)=c|u|^{p^{*}-2} u+k(x)|u|^{q-2} u+g(x, u), & x \in \Omega \\ u=0, & x \in \partial \Omega .\end{cases}
$$


Corollary 3.6 Suppose the parameters $1 \leq \alpha<p$ and $q<p^{*}, c>0$, the functions $k(x) \in$ $C(\bar{\Omega})$ with $k(x) \geq k^{*}>0$, and $g(x, u) \in C(\bar{\Omega} \times \mathbf{R})$ with $g(x, 0)=0, g(x, u) u \geq 0$. Moreover,

$$
q>\left\{\begin{array}{ll}
p^{*}-\frac{N}{N-1} & \text { if } \alpha \leq \frac{N(p-1)}{(N-1)}, \\
\frac{N \alpha}{N-p} & \text { if } \alpha \geq \frac{N(p-1)}{(N-1)},
\end{array} \quad \lim _{u \rightarrow \infty} \sup _{x \in \Omega} \frac{|g(x, u)|}{|u|^{p^{*}-1}}=0, \quad \limsup \sup _{x \in \Omega} \frac{g(x, u)}{|u|^{p-2} u}<S .\right.
$$

Then problem (3.2) possesses a positive solution in $C^{1, \alpha}(\Omega)(\alpha \in(0,1))$.

Proof Letting $m_{1}=0$ and $m_{2}=\alpha$ in Lemma 3.1, and combining Lemma 3.2, Lemma 3.3 and Theorem 1.3, we can derive the conclusion.

\section{Competing interests}

The authors declare that they have no competing interests.

\section{Authors' contributions}

ZY brought out the problem and gave the proof of the existence of a nontrivial solution. GD suggested that the generalized C-C principle could be applied to this problem and proved the non-trivial solution could be a positive solution. HY improved the regularity of the solution and checked all of the proof.

\section{Author details}

${ }^{1}$ School of Mathematics Science, South China Normal University, Guangzhou, 510631, China. ${ }^{2}$ School of Mathematics and Computer Science, Guangdong University of Business Studies, Guangzhou, 510320, China.

\section{Acknowledgements}

The project is supported by National Natural Science Foundation of China (No. 11271143, 10901060, 10971073), the Natural Science Foundation of Zhejiang Province (No. Y6110775, Y6110789).

\section{Received: 14 May 2012 Accepted: 13 September 2012 Published: 2 October 2012}

\section{References}

1. Brezis, H, Louis, N: Positive solutions of nonlinear elliptic equations involving critical sobolev exponents. Comm. Pure Appl. Math. 36, 437-477 (1983)

2. Chen, Z-H, Shen, Y-T: Some existence results of solutions for $p$-Laplacian. Acta Math. Sci., Ser. B Engl. Ed. 23, 487-496 (2003)

3. Halidias, N: Existence theorems for nonlinear elliptic problems. Arch. Inequal. Appl. 6(3), 305-323 (2001)

4. Song, C-X, Wen, P-X: Eigenvalue problems for $p$-Laplacian functional dynamic equations on time scales. Adv. Differ. Equ. 2008, Article ID 879140 (2008)

5. Xuan, B-J: The solvability of quasilinear Brezis-Nirenberg-type problems with singular weights. Nonlinear Anal. TMA 62, 703-725 (2005)

6. Zhu, X-P: Nontrivial solution of quasilinear elliptic equations involving critical Sobolev exponent. Sci. Sin., Ser. A (3) 16, 225-237 (1988)

7. Lion, P: The concentration-compactness principle in the calculus of variation, the limit case, part 1. Rev. Mat. Iberoam. 1(1), 145-201 (1985)

8. Lion, P: The concentration-compactness principle in the calculus of variation, the limit case, part 2. Rev. Mat. Iberoam. $1(2), 45-121(1985)$

9. Fu, H-Z, Shen, Y-T, Yang, J: p-mean curvature operator with critical exponent. Chin. Q. J. Math. 21, $511-521$ (2006)

10. Yang, Z, Geng, D, Yan, H-W: Some generalizations to the concentration-compactness principle. Southeast Asian Bull. Math. 33, 597-606 (2009)

11. Yang, Z, Geng, D, Yan, H-W: Three solutions for singular p-Laplacian type equations. Electron. J. Differ. Equ. 2008, 61 (2008)

12. Du, K, Meng, Q-X: A revisit to theory of super-parabolic backward stochastic partial differential equations. Stoch Process. Appl. 120(10), 1996-2015 (2010)

13. Du, K, Zhang, Q: Semi-linear degenerate backward stochastic partial differential equations and associated forward backward stochastic differential equations. http://arxiv.org/abs/1109.0672 (2011)

14. Geng, D, Yang, Z: On the weakly continuity of $p$-Laplace operator in quasilinear elliptic equations with critical growth J. South China Norm. Univ., Nat. Sci. Ed., 2003(3), 10-13 (2003)

15. Peter, T: Regularity for a more general class of quasilinear elliptic equations. J. Differ. Equ. 51, 126-150 (1984)

16. Marcelo, M: Strong maximum principles for supersolutions of quasilinear elliptic equations. Nonlinear Anal. 37, 431-448 (1999) 